\title{
TAGUNG
}

\section{Auf dem Weg zum Mehrebenenparlamentarismus? Zukünftige Funktionen von Parlamenten im europäischen Integrationsprozess}

\author{
Ellen Schneider*
}

Auf der wissenschaftlichen Tagung des Arbeitsbereichs Vergleichende Politikwissenschaft und Europäische Integration der Universität Tübingen und des Arbeitskreises Europäische Integration e.V., die von Gabriele Abels und Annegret Eppler am 26. und 27. Mai 2011 in Tübingen durchgeführt wurde, befassten sich Vertreter aus Wissenschaft und Praxis aus sehr unterschiedlichen Perspektiven mit der Metapher, Mehrebenenparlamentarismus'. Die Konsequenzen aus der Stärkung der Parlamente in der Europäischen Union durch den Vertrag von Lissabon und das sogenannte Lissabon-Urteil des Bundesverfassungsgerichts wurden für die Parlamente aller Ebenen des europäischen Mehrebenensystems sowohl hinsichtlich ihrer verfassungsrechtlichen Stellung als auch mit Blick auf ihre tatsächlichen Aktivitäten herausgearbeitet. Schwerpunkte der Diskussionen bildeten dabei die folgenden Fragen: Was ist unter Mehrebenenparlamentarismus $\mathrm{zu}$ verstehen? Inwiefern verändert dieses Konzept die Funktionen von Parlamenten auf verschiedenen Ebenen des politischen Systems? Ist Mehrebenenparlamentarismus in der Lage, zu einer Verringerung des Demokratiedefizits der Europäischen Union beizutragen?

Stand und Ausblick der Parlamentarisierung der Europäischen Union

Schwerpunkt des ersten Panels war die Einordnung der Parlamentarisierungsstrategie der Europäischen Union, die im Vertrag von Lissabon ihren Höhepunkt gefunden hat. Nach

\section{Auf dem Weg zum Mehrebenen- parlamentarismus? Zukünftige Funktionen von Parlamenten im europäischen Integrationsprozess}

Wissenschaftliche Tagung des Arbeitsbereichs Vergleichende Politikwissenschaft und Europäische Integration der Universität Tübingen und des Arbeitskreises Europäische Integration e.V.

Die Tagung wurde von der Europäischen Kommission finanziell unterstützt.

Tübingen, 26.-27. Mai 2011

Begrüßung

Prof. Dr. Gabriele ABELS, Institut für Politikwissenschaft, Universität Tübingen

Dr. Annegret EPPLER, Ass. iur., Institut für Politikwissenschaft, Universität Tübingen

Parlamente und ihre Herausforderungen

Dr. Andreas MAURER, Stiftung Wissenschaft und Politik, Berlin

Prof. Dr. Berthold RITTBERGER, Lehrstuhl für Politische Wissenschaft und Zeitgeschichte, Fakultät für Sozialwissenschaften, Universität Mannheim Rainer WIELAND, MdEP, Vizepräsident des Europäischen Parlaments, Stuttgart

Nationale Parlamente unter europäischem Einfluss

Dr. Katrin AUEL, Frankfurter Institut für Transformationsstudien, Europa-Universität Viadrina, Frankfurt an der Oder

Prof. Dr. Timm BEICHELT, Professur für „Europa-Studien“, Europa-Universität Viadrina, Frankfurt an der Oder

Dr. des. Martin GROßE HÜTTMANN, Institut für Politikwissenschaft, Universität Tübingen

* Ellen Schneider, M.A., Akademische Mitarbeiterin, Mannheimer Zentrum für Europäische Sozialforschung, Mannheim. 
einer Definition des Begriffs Mehrebenenparlamentarismus als Stärkung der Parlamente auf allen Ebenen in EU-Entscheidungsprozessen gab Andreas Maurer im ersten Vortrag einen Überblick über die im Laufe der europäischen Integration geänderten Funktionen des Europäischen Parlaments sowie der nationalen Parlamente. Anschließend überprüfte er den Wandel der Funktionen auf ihren empirischen Gehalt. Während die Politikgestaltungs- und Kontrollfunktionen beider parlamentarischer Ebenen als gestärkt interpretiert werden könnten, liege eine normative wie empirische Vernachlässigung der Integrationsfunktion gegenüber den Bürgern vor. Insgesamt machte er zudem deutlich, dass gerade die nationalen Parlamente die erworbenen Mitwirkungsrechte oft nicht in konkretes Handeln umsetzten und sich häufig durch die Regierung instrumentalisieren ließen. Berthold Rittberger setzte sich mit der Frage auseinander, wie sich die durchaus konfliktträchtige zunehmende Institutionalisierung des Europäischen Parlaments erklären lasse, die in einer kontinuierlichen Funktions- und Kompetenzausweitung deutlich werde. Hierbei stellte er fest, dass die Rolle des Europäischen Parlaments beim Abbau des Demokratiedefizits zunehmend weniger umstritten gewesen sei. Somit besäßen nicht regulative, sondern normative und vor allem kognitivkulturelle Faktoren das höchste Erklärungspotenzial für die Stärkung des Europäischen Parlaments. Rainer Wieland machte anschlieBend aus seiner Sicht als Mitglied und Vizepräsident des Europäischen Parlaments deutlich, dass sowohl bei der Zusammenarbeit im Sinne eines wirklichen Mehrebenensystems als auch bei der Ausfüllung parlamentarischer Funktionen - und hier insbesondere der Einbindung der Bürger - noch Verbesserungspotenzial bestehe. Zugleich warnte er mit Blick auf den Zielkonflikt zwischen Input- und Output-Legitimation davor, den diskretionären Spielraum zuständiger Beamten zu stark zu beschneiden, da dies zu einer Selbstentmachtung als Mitgliedstaat führe und sogenannte ,package deals' dann nicht mehr möglich seien. In der anschließenden Diskussion
Podiumsdiskussion: Zur Zukunft des Parlamentarismus in der EU

Moderation:

Prof. Dr. Gabriele ABELS, Universität Tübingen

\section{Diskutanten:}

Daniel LEDE ABAL, MdL, Baden-Württemberg, Tübingen

Dr. Katrin AUEL, Europa-Universität Viadrina, Frankfurt an der Oder

Peter HAUK, MdL, Fraktionsvorsitzender der CDU-Fraktion, Baden-Württemberg, Mosbach

Dr. Andreas MAURER, Stiftung Wissenschaft und Politik, Berlin

Alexandra THEIN, Mitglied des Europäischen Parlaments, Berlin

Ministerialrat Dr. Sven VOLLRATH, Leiter des Europareferats des Deutschen Bundestages, Berlin/Brüssel

\section{Fallbeispiel Deutschland}

Prof. Dr. Matthias NIEDOBITEK, Institut für Europäische Studien, Technische Universität Chemnitz

Ministerialrat Dr. Sven VOLLRATH, Deutscher Bundestag, Berlin/Brüssel

Ministerialrätin Ute MÜLLER, Leiterin des Sekretariats des Ausschusses für Fragen der Europäischen Union, Geschäftsführerin der Europakammer, Bundesrat, Berlin

\section{Problemdimension der Parlamentarisierung}

Prof. Dr. Carina SPRUNGK, Otto-Suhr-Institut für Politikwissenschaft, Freie Universität Berlin

Prof. Dr. Sabine KROPP, Lehrstuhl für Politikwissenschaft, Deutsche Hochschule für Verwaltungswissenschaften, Speyer

Carmen PREISING, Stellvertretende Referatsleiterin, Generalsekretariat der Europäischen Kommission, Brüssel

Die Rolle zweiter Kammern und subnationaler Parlamente

Univ.-Prof. Dr. Anna GAMPER, Institut für Öffentliches Recht, Staats- und Verwaltungslehre, Universität Innsbruck

Prof. em. Dr. Frank DELMARTINO, Fakultät für Sozialwissenschaften, Katholische Universität Leuven; Visiting Professor am Europakolleg Brügge

Prof. Dr. Gabriele ABELS, Institut für Politikwissenschaft, Universität Tübingen 
wurde deutlich, dass die Mehrheit der Teilnehmer ein Demokratiedefizit in der Europäischen Union als gegeben ansieht. Hier wurde neben der Strategie der Parlamentarisierung insbesondere die Herausforderung für die verschiedenen Parlamente hervorgehoben, die Bürger deutlicher in europäische Entscheidungsprozesse einzubeziehen. Dazu sei auch eine Veränderung der medialen Berichterstattung nötig.

\section{Problemdimensionen der Europäisierung}

Im zweiten Panel gab zunächst Katrin Auel einen kritischen Überblick über die Literatur zur Europäisierung parlamentarischer Demokratien. Dabei bemängelte sie den engen Fokus der existierenden Forschung: Häufig werde sich auf die Entwicklung formaler Rechte und Kompetenzen sowie die Legislativ- und Kontrollfunktion beschränkt. Hinsichtlich der Debatte zwischen dem Verständnis des Parlaments als individuelle oder in ein Mehrebenensystem eingebettete Institution hob sie das von Ben Crum und John E. Fossum entwickelte Konzept des „Multilevel Parliamentary Field" als vielversprechend hervor. Dieser Ansatz fand auch in der anschließenden Diskussion Anklang bei den Teilnehmern. Dieses momentan noch rein heuristische Konzept sei in der Lage, nicht mehr nur die Summe der einzelnen (Parlaments-)Teile darzustellen, sondern die Beziehungen und Legitimitäten über drei Formen parlamentarischer Repräsentation zu analysieren. Timm Beichelt betrachtete in seinem Vortrag zur Rolle nationaler Exekutiven im Mehrebenenparlamentarismus die Parlamentsfunktionen und analysierte, inwiefern diese unter Einbeziehung nationaler Regierungen verwirklicht sind. Hierbei stellte er fest, dass die Regierungen als europäischer Primärgesetzgeber gelten können. Außerdem verhielten sie sich wie quasi-parlamentarische Akteure, mit dem Unterschied, dass ihre repräsentative Funktion sehr viel stärker auf Partikularinteressen bezogen sei. Die Legitimität dieses exekutiven Parlamentarismus' lasse sich dabei nicht durch eine „beste normative Lösung“ erhöhen, da je nach Interpretationsmodell des
Parlamentarische Vernetzung im Mehrebenenparlamentarismus

Dr. Annegret EPPLER, Ass. iur., Universität Tübingen

Univ.-Prof. Dr. Jürgen MITTAG, Institut für Europäische Sportentwicklung und Freizeitforschung, Deutsche Sporthochschule Köln

Dr. Henrik SCHELLER, Wissenschaftlicher Mitarbeiter am Lehrstuhl Politik und Regieren in Deutschland und Europa, Universität Potsdam

Abschlussdiskussion und Schlussworte

Prof. Dr. Gabriele ABELS, Universität Tübingen

Mehrebenenparlamentarismus (liberaler Nationalstaat und Intergouvernementalismus einerseits oder Mehrebenensystem und Supranationalismus andererseits) unterschiedliche Verhaltensstrategien zur Legitimierung beitrügen. Martin Große Hüttmann betonte in seinem Vortrag den Unterschied zwischen geschriebener und gelebter Verfassung. So gebe es auf der verfassungsrechtlichen Systemebene eine Demokratisierung oder auch Parlamentarisierung. Auf der Handlungsebene müsse man - gerade vor dem Hintergrund der Eurokrise - eher von Präsidialisierung sprechen. Die Gefahr einer grundlegenden Transformation hin zu einer europäischen Wirtschaftsregierung, die in Krisenzeiten ihre Regelungen durch Gipfeldemokratie trifft, wurde auch in der anschließenden Diskussion vielfach aufgegriffen und kritisiert.

Podiumsdiskussion zu den Herausforderungen des europäischen Mehrebenenparlamentarismus

Tenor der Podiumsdiskussion am Ende des ersten Tages war, dass trotz der Verbesserungen durch den Vertrag von Lissabon ein Legitimationsproblem im Parlamentarismus der Europäischen Union existiere. Dessen Umfang und mögliche Strategien um es zu verringern, waren hingegen umstritten. Die mangelnde Einbindung der Bürger sowie die fehlende Öffentlichkeit waren dabei Hauptkritikpunkte. Während einige diese Mängel durch einen verstärkten Wettkampf zwischen Regierung und Opposition und generell eine 
stärkere Rolle der Parteien beheben wollten, lobten andere die konsensuale Entscheidungsfindung in der Europapolitik auf den verschiedenen politischen Ebenen oder sahen gerade im Repräsentations- und Nominationsmonopol der Parteien Gründe für die eher geringe Akzeptanz der Europäischen Union durch die Bürger. Die Wichtigkeit eines frühzeitigen Eingreifens und der Aufbau eines parlamentarischen Kontaktnetzes wurden mehrfach betont; letzteres gerade auch, um zu verhindern, dass verschiedene Ebenen gegeneinander ausgespielt werden. Hier wurde deutlich, dass die ohnehin schon hoch komplexe Aufgabe der Vernetzung aller Ebenen des europäischen Systems aufgrund der Verschiedenheit der Akteure beziehungsweise ihrer Interessendivergenzen und eines ,,permanenten Drehtüreffekts" mit neuen Akteurskonstellationen zusätzlich erschwert werde. Die Verwaltungen sind hier nach Auffassung der Diskussionsteilnehmer aufgrund ihrer Kontinuität im Vorteil. Darüber hinaus gelinge eine Vernetzung nur einzelnen ressourcenstarken Mitgliedstaaten beziehungsweise Regionen sowie manchen Parteien. Insgesamt wurde bei der Podiumsdiskussion deutlich, dass nur eine Mischung verschiedener repräsentativ-demokratischer sowie partizipatorischer Instrumente in der Lage ist, die demokratische Legitimation zu festigen. Vorschläge bezogen sich dabei zum einen auf eine weitere Parlamentarisierung sowie eine Wahlreform, etwa durch die Errichtung eines gemeinsamen europäischen Wahlkreises oder die Einführung eines Vorzugsstimmenmodells. Zum anderen wurde die mit dem Vertrag von Lissabon eingeführte Europäische Bürgerinitiative als geeignetes Mittel genannt, um über direkt-demokratische Beteiligung mehr Bürgernähe zu erreichen.

Der Vertrag von Lissabon im Alltag: Auswirkungen für nationalstaatliche Institutionen in Deutschland

Am zweiten Konferenztag erläuterte zunächst Matthias Niedobitek die Auswirkungen des Lissabon-Urteils des Bundesverfas- sungsgerichts für die Gesetzgeber, bevor Sven Vollrath die Umsetzung dieser neuen Mitwirkungsrechte für den Bundestag und Ute Müller für den Bundesrat aufzeigten. Niedobitek machte dabei deutlich, dass die detaillierten Vorgaben für die Begleitgesetzgebung, die als „Misstrauen“ gegenüber dem Gesetzgeber interpretiert wurden, durchaus berechtigt seien, da der Bundestag sich in seiner Kontrollfunktion gegenüber der Regierung oft selbst einschränke. Die dem Bundestag übertragene Integrationsverantwortung beschränke sich auf den konstitutionellen Bereich und beziehe sich nicht auf das ,Alltagsgeschäft ${ }^{\star}$. Nach seiner Auffassung wurde mit diesem Urteil das Legitimationsdefizit der Europäischen Union nicht verringert, sondern nur vor einer Vergrößerung bewahrt. Sowohl Vollrath als auch Müller unterschieden zwischen dem in Bezug auf die quantitative Umsetzung als „Sonntagsgesetz" einzuordnenden Integrationsverantwortungsgesetz und dem wichtigeren jeweiligen Zusammenarbeitsgesetz. Die europapolitischen Aktivitäten und die Wahrnehmung der Beteiligungsrechte des Bundestags sind laut Vollrath deutlich gestiegen. Müller zeigte für den Bundesrat, dass er eine neue Rolle auf europäischer Ebene einnehme, mit dem Instrument der Subsidiaritätskontrolle aber noch offensiver umgehen könne. Die von beiden kritisierte Entparlamentarisierung in Hinblick auf verschiedene, im Rahmen der Weltwirtschaftskrise auf intergouvernementaler Ebene getroffene Entscheidungen wurde auch in der anschließenden Diskussion gerügt. Des Weiteren wurde dargelegt, dass Parlamentarier ihre eigene Regierung auf europäischer Ebene nicht schwächen wollen und im Bundestag gerade erst begonnen wird, die unterschiedlichen Positionen zwischen Regierungs- und Oppositionsparteien kenntlich zu machen. Zudem sei die Vernetzung beider Parlamente noch ausbaufähig.

Konsequenzen der Europäisierung aus unterschiedlicher Perspektive

Im anschließenden Panel beschäftigten sich die Vortragenden mit den Auswirkungen ei- 
ner zunehmenden Europäisierung für die nationalen Parlamente in der Europäischen Union, gerade auch vor dem Hintergrund des Vertrags von Lissabon. Carina Sprungk konzentrierte sich vor allem auf die Kontrollfunktion nationaler Parlamente und stellte fest, dass die Formalisierung der Informationsbeschaffung und die damit verbundene Möglichkeit der ex-post Kontrolle zumindest eine indirekte Stärkung dieser Parlamentsfunktion bedeuten. Insgesamt habe es seit Inkrafttreten des Vertrags von Lissabon zudem eine leichte Steigerung der Interaktion mit Vertretern verschiedener Institutionen der Europäischen Union gegeben; ob dies wirklich zu einer stärkeren Regierungskontrolle führt, sei jedoch unklar. Mit der Subsidiaritätskontrolle stehe den nationalen Parlamenten zudem eine - allerdings ,negative“ - Gesetzgebungsfunktion zur Verfügung, deren effektive Nutzung aber ebenfalls stark von inter-parlamentarischen Kooperationen abhänge. Das Interesse von Sabine Kropp galt der Alltagseuropäisierung in Bezug auf Rollenorientierungen und Rollenerwartungen. Sie verglich dabei den Einfluss von Rollen auf das Verhalten von Akteuren der Legislative und der Ministerialbürokratie in Deutschland, Schweden und Ungarn für die beiden unterschiedlich stark europäisierten Politikfelder Umwelt- und Sozialpolitik. Hierbei stellte sie fest, dass es tatsächlich zu einer Europäisierung der Rollen kommt und das Expertenparlament einen Transformationsprozess durchläuft. Gerade Abgeordnete mit europäisierten Rollenorientierungen wollten durch Nichtregierungsorganisationen oder Parteien bereitgestellte, politisch bewertete Informationen; die Ministerialbürokratie sei ihnen hier weniger wichtig. Allerdings gebe es eine Akzeptanz der Rollenteilung mit der Ministerialbürokratie, eine legislative Detailsteuerung sei nach Meinung eines Teils der Abgeordneten nicht mehr nötig. Im letzten Vortrag dieses Panels schilderte Carmen Preising den Umgang der nationalen Parlamente mit den neuen Rechten des Vertrags von Lissabon aus der Perspektive der Europäischen Kommission. Sie beschränkte sich dabei auf den Frühwarnmecha- nismus und hielt fest, dass obwohl der für eine Subsidiaritätsrüge nötige Schwellenwert der Beteiligung nationaler Parlamente bisher noch nie erreicht wurde, dennoch ein politischer Dialog über Gesetzesvorschläge entstanden sei. Die nationalen Parlamente hätten dabei große Fortschritte gemacht und zudem ihre Reaktionsgeschwindigkeit auf Vorhaben der Europäischen Union deutlich erhöht. Die größte Herausforderung bleibe die Koordination der nationalen Parlamente, die die Europäische Kommission zu unterstützen versuche. Auch in der anschließenden Diskussion wurde deutlich, dass der von allen als wichtig empfundene politische Dialog der verschiedenen Ebenen erst am Anfang stehe, die nationalen Parlamente sich stärker vernetzen müssten und der erste Fall einer erfolgreichen Subsidiaritätsrüge mit Spannung erwartet werde.

\section{Gewinner des Vertrags von Lissabon? Zweite Kammern und subnationale Parlamente}

Aus Sicht von Anna Gamper kam es durch die Regelungen des Vertrags von Lissabon und die Gleichbehandlung beider Kammern beim Stimmrecht, wenn überhaupt, nur zu einer leichten Stärkung der zweiten Kammern. Diese könne sich zudem je nach bikameralem System deutlich unterscheiden, da es (mit Ausnahme Belgiens) keine Definition zweiter Kammern gebe und so die einzelnen Mitgliedstaaten einen weiten Ermessensspielraum besäßen. Auch in Hinblick auf die Frage, ob die neuen Rechte zur Legitimierung der Europäischen Union beitragen können, blieb sie skeptisch und sah allenfalls einen indirekten Beitrag. Das Subsidiaritätsprinzip gebe als Primärrecht zwar eine gewisse Legitimation, sei aber kein positives oder gestalterisches Element, sondern habe eher einen reagierenden, kontrollierenden und misstrauischen Charakter. Frank Delmartino machte gleich zu Beginn seines Vortrags zu den sieben Parlamenten Belgiens auf föderaler und subnationaler Ebene deutlich, wie wichtig die Unterscheidung zwischen Verfassungsrecht und Verfassungspraxis sei. Insgesamt lobte er die 
gute Zusammenarbeit der Gemeinschaften, Regionen und deren Parlamente mit dem Föderalstaat in europäischen Angelegenheiten. Er war allerdings auch der Ansicht, dass die Parlamentarier die Vorgehensweise auf mehreren Ebenen noch nicht verinnerlicht hätten. Die von ihm getroffene Unterscheidung zwischen dem Konzept des Mehrebenenparlamentarismus, das nach seiner Auffassung auf demokratische Legitimität zuläuft, und dem Konzept der ,multilevel governance', das eher auf Problemlösungskapazitäten fokussiere, fand auch in der anschließenden Diskussion Zustimmung bei den Teilnehmern. In ihrem abschließenden Beitrag zur Europa-Arbeit der deutschen Landtage nach ,Lissabon“ stellte Gabriele Abels sowohl Kontinuität als auch Wandel fest. So bleibe die Exekutivabhängigkeit deutscher Landtage bestehen und die dokumentenbasierte Kontrolle dominiere, während die mandatsbasierte Strategie bisher nur im Landtag von Baden-Württemberg eingeführt worden sei. In Bezug auf diese Strategie der Bindung der Landesregierung in bestimmten Fällen wurde in der abschließenden Diskussion deutlich, dass umstritten ist, ob die mandatsbasierte Strategie verfassungskonform sei, und es darauf ankomme, wie dieses Gesetz im ersten Konfliktfall in der Praxis ausgelegt werde. Abels sowie die Diskussionsteilnehmer betonten zudem, dass die Nutzung der neuen Kontroll- und Mitwirkungsrechte stark von der Ressourcenausstattung abhänge, deren Mangel nur zum Teil durch Kooperationen ausgeglichen werden könne. Abschließend wurde die Artikulationsfunktion der Landtage aufgrund der größeren Bürgernähe der subnationalen Ebene hervorgehoben.

\section{Mehrebenenvernetzung als Möglichkeit zur Steigerung der Legitimation?}

Annegret Eppler machte im ersten Vortrag dieses Panels die komplexe Aufgabe der horizontalen, vertikalen und über parlamentarische Akteure hinausgehenden Zusammenarbeit der Parlamente deutlich, vor der die verschiedenen Parlamente im europäischen Mehrebenensystem stünden. Hierbei stellte sie fest, dass man sich in der Zusammenarbeit nach wie vor am Anfang befinde und die Koordination extrem schwierig sei. Die nationalen Parlamente sah sie als weiter fortgeschritten als die subnationalen, stellte insgesamt aber starke Abhängigkeiten vor allem finanzieller Art fest. Die informelle Zusammenarbeit sei - auch wenn diese häufig nicht strategisch erfolge - weiter fortgeschritten als die formell kodifizierte. Insgesamt sah sie die Kontrollfunktion ebenenübergreifend im Vordergrund und fragte sich, inwiefern dies gemäß dem Politikverflechtungsansatz problematisch werden könne. Abschließend stellte sie fest, dass sich die Parlamentarier der verschiedenen Ebenen bereits allein aufgrund mangelnder Ressourcen noch nicht vom Souverän zum Lobbyisten gewandelt hätten, sich aber dennoch die Frage stelle, inwiefern das , multilevel governance'-System mit seiner Output-Orientierung die input-orientierten Parlamente präge. Die während der Tagung vielfach geforderte Stärkung politischer Parteien als Bindeglied verschiedener Ebenen wurde von Jürgen Mittag für die Beziehungen zwischen nationaler und europäischer Ebene einer Bestandsaufnahme unterzogen. Die nationalen Parteien bleiben seiner Ansicht nach insgesamt nach wie vor vorrangig auf den nationalen Kontext ausgerichtet, während dies bei den Fraktionen im Europaparlament anders sei und auch bei den europäischen Parteien zumindest im Ansatz eine zunehmende Vernetzung entstehe. Die Einordnung politischer Parteien als Bindeglieder verschiedener Ebenen lasse sich vorläufig noch nicht vornehmen; zudem bleibt für ihn die Frage bestehen, inwiefern Netzwerkparteien und funktionale Parteien als Legitimationsbeschaffer fungieren können. In der Diskussion wurde auch deutlich, dass die mehrfach geforderte Politisierung durch Parteien nach wie vor umstritten bleibt, da es neben herausragenden und kompetitiven Ereignissen wie der Wahl des Kommissionspräsidenten auch sehr technische Debatten gebe. Henrik Scheller machte im letzten Vortrag der Tagung die Notwendigkeit eines Wandels des parlamentarischen Selbstverständnisses hinsichtlich neuer Kom- 
munikationstechnologien deutlich. Bei seiner Analyse der Nutzung sozialer Medien durch die verschiedenen Parlamente im europäischen Mehrebenensystem zeigte er auf, dass es keinerlei virtuelle Vernetzung zwischen dem Europäischen Parlament und den nationalen und subnationalen Parlamenten der Mitgliedstaaten gibt. Letztere seien in den sozialen Netzwerken sogar überhaupt nicht vertreten. Diese mangelnde Nutzung virtueller Vernetzungsmöglichkeiten im europäischen Mehrebenensystem wurde auch in der anschließenden Diskussion kritisiert. Trotz der mit neuen sozialen Medien einhergehenden Probleme, wie der Unterrepräsentation bestimmter Gruppen, bestand Einigkeit, dass sich hier eine wirkliche Möglichkeit zur interparlamentarischen Kommunikation biete.

Fazit: Die Spannung zwischen Input- und Output-Legitimation bleibt

In der abschließenden Diskussion wurden verbleibende theoretische und methodologische Herausforderungen konstatiert, vor denen die Wissenschaft nach Meinung der Teilnehmer steht. Eine stärkere Verknüpfung der Parlamentarismus- und Europaforschung wurde dabei als notwendig erachtet und der Kontakt und Dialog zwischen Wissenschaftlern und Vertretern der Praxis aller Arbeitsebenen als fruchtbar empfunden. In Bezug auf das hinter der Metapher Mehrebenenparlamentarismus stehende Konzept wurde postuliert, dass die Erweiterung der separaten Betrachtung einzelner Parlamente und ihrer Funktionen um eine Analyse der Beziehungen zwischen den verschiedenen Ebenen im europäischen Mehrebenensystem essenziell sei. Dadurch könne auch eine theoretische Einordnung und die Beantwortung der Frage gelingen, ob die Parlamente in der Europäischen Union auf dem Weg zu einem input-orientierten Mehrebenenparlamentarismus oder dem eher output-orientierten Multi-Level-Governance-System sind. Es wurde darüber hinaus angemerkt, dass sich die Europaforschung womöglich aufgrund des Phänomens des Mehrebenenparlamentarismus von tradierten Vorstellungen der Legitimation abwenden und neue wissenschaftliche Legitimationskonzepte entwickeln müsse. Mit Blick auf die Funktionen von Parlamenten wurde in der Diskussion deutlich, dass die Möglichkeit, unterschiedliche Ebenen schwerpunktmäßig mit unterschiedlichen parlamentarischen Funktionen auszustatten, noch nicht ausgeschöpft sei. Bisher gebe es eine Dominanz von Kontrollfunktionen mit der immanenten Gefahr der Blockade durch zu starke Politikverflechtung, dabei sei im Gegensatz dazu eine Stärkung der Artikulationsfunktion gerade für die subnationale Ebene sehr vielversprechend. Bezüglich des Demokratiedefizits blieb bei den Teilnehmern der Tagung das richtige Verhältnis von Input-und Output-Legitimation nach wie vor unklar: Eine Stärkung der Parlamente wurde festgestellt; der damit einhergehende Zielkonflikt zwischen dem gewünschten $\mathrm{Ma} ß$ von Kontrolle und Freiraum der zuständigen exekutiven Institutionen wurde allerdings ebenfalls angemerkt. 